\title{
Effects of Inter-Subject Variability and Vibration Magnitude on Vibration Transmission to Head during Exposure to Whole-Body Vertical Vibration
}

\author{
Milk Desta, V. Huzur Saran and Suraj P. Harsha \\ Vehicle Dynamics Lab., Mechanical and Industrial Engineering Department \\ Indian Institute of Technology Roorkee, India
}

\begin{abstract}
In this paper, the effect of inter-subject and intra-subject variabilities on transmission of vibration through seated human subjects is discussed using experimental results. The experimental study targeted three representative postures (backrest, erect, and forward lean on table) while performing sedentary activities and under three magnitudes $\left(0.4,0.8,1.2 \mathrm{~m} / \mathrm{s}^{2} \mathrm{rms}\right)$ of vertical vibration. The frequency range considered is $1 \mathrm{~Hz}-20 \mathrm{~Hz}$ as representative of those likely prevailing in wide range of vehicles. The data sets are investigated in terms of STH (seat-to-head) and BTH (back support-to-head) transmissibilities and phase differences, and respective coherences under the magnitudes and postures undertaken. In addition to determining the effect of different frequencies, subjective readings were collected at vertical backrest support postures at representative frequencies using the Borg CR 10 comfort scale. The responses show significant variations in transmissibility and phase among all of the subjects. In all postures, the mean STH transmissibility increases with increasing vibration magnitude at body resonance frequency, which lies approximately between $4.5 \mathrm{~Hz}$ and $6 \mathrm{~Hz}$. Resonance in STH transmissibility of erect and forward lean on table posture visibly tends to shift to a lower frequency with increasing vibration magnitude. The subjective reading obtained, in terms of discomfort level, match with experimental data sets and provides evidence that human body resonance frequency or discomfort zone is around $5 \mathrm{~Hz}$. Therefore, it might be concluded that the inclusion of vibration magnitude, posture, and inter-subject variabilities in the prediction of seat biodynamic response is essential. The development of biodynamic models and design of seat should include the variation of STH and BTH transmissibility and phase in different possible postures under different vibration magnitude.
\end{abstract}

\section{INTRODUCTION}

The study of human response to vibration in a sitting posture is very important to reduce the effects of vibration to human health, activity, and performance. Research on the vibration effects of seated subjects has indicated that the side effects could be very harmful and in some cases lead to permanent injuries. ${ }^{1}$ Some results have suggested that lower back pain is a result of continuous exposure to vibration, ${ }^{2}$ and occurs more frequently among vehicle drivers than in representative control groups. ${ }^{3}$ As traveling increases, the driver is more exposed to vibration that originates primarily from the interaction between the road profile and the vehicle. Therefore, in recent years people have become more concerned with vibration and are seeking a more comfortable environment. Indeed, a seat with optimum dynamic properties is one that minimizes the unwanted vibration responses of humans in the relevant vibration environment. The three important factors which determine the seat dynamic efficiency are vibration environment, seat dynamic response, and response of the human body. To achieve better and comfortable vibration condition, that is, to reduce vibration to massage movement, there is a pressing need to study and identify whole-body vibration discomfort zone in vehicle seats. By doing so, criteria for better ride comfort can be determined so that seat and vehicle systems are designed optimally.

The study of human response to vibration has been the topic of interest over the years and a number experimental and ana- lytical studies were established in different vibration environments. The transmissibility of the human body reflects the various biodynamic responses of the body, particularly those between the point at which the vibration enters the body (e.g., on a seat) and the point at which the vibration is measured on the body (e.g., on the head). The transmissibility, therefore, gives some information on the biodynamic system. It has sometimes been assumed that the resonances reflected in, for example, the STH transmissibility indicate frequencies at which injury, discomfort, or interference with activities are most likely to happen.

The effect of a sitting posture on the apparent mass of a subject (i.e., the ratio of the force to the acceleration as a function of vibration frequency) has previously been reported. ${ }^{4-6}$ Although Miwa ${ }^{4}$ has stated that "no clear difference was reckoned to exist" between sitting relaxed or erect, his data show a small effect that is consistent with results from Fairley and Griffin. ${ }^{5}$ Kitazaki and Griffin ${ }^{6}$ suggest that the resonance frequency of the human body is higher in a more erect sitting posture. Fairley and Griffin ${ }^{5}$ have investigated eight subjects who sat in four postures (normal, erect, backrest contact, and tense) and generally exhibited higher resonance frequencies for the erect and tense postures compared to the normal posture. Kitazaki and Griffin ${ }^{6}$ have shown an increase in the mean resonance frequency from $4.4 \mathrm{~Hz}$ to $5.2 \mathrm{~Hz}$ when eight subjects sat in slouched and erect postures. The change can be described as a stiffening effect with erect postures.

The literature shows that some variables can have large ef- 